\title{
Aortitis sifilítica: a propósito de un caso del sur colombiano
}

\author{
Syphilitic aortitis: about a case in South Colombia
}

\author{
Diego J. Alvis-Peña ${ }^{1 *}$, Carlos H. Calderón-Franco², Julieth Gonzales-Cerón ${ }^{3}$, María P. Alvis-Peña ${ }^{4}$ y \\ Yexica A. Rodríguez-Rodríguez ${ }^{5}$
}

${ }^{1}$ Servicio de Medicina Interna e Investigación, Hospital Universitario Hernando Moncaleano Perdomo, Neiva, Huila; ${ }^{2}$ Servicio de Medicina Interna e Investigación, Clínica Mediláser, Florencia, Caquetá; ${ }^{3}$ Servicio de Medicina Interna e Investigación, Hospital Universitario Hernando Moncaleano Perdomo, Neiva, Huila; ${ }^{4}$ Unidad Académica, Universidad Surcolombiana, Neiva, Huila; ${ }^{5}$ Unidad Académica, Fundación Universitaria Navarra, Neiva, Huila. Colombia

\section{Resumen}

Se expone el caso de una paciente de 73 años procedente y residente de Florencia, Caquetá (Colombia), con antecedentes de hipertensión arterial, que ingresa a un hospital de alta complejidad por un cuadro clínico de 3 meses de evolución consistente en dolor torácico tipo opresivo en la región anterior izquierda del tórax, no irradiado, de intensidad 8/10. En el ecocardiograma se evidencia enfermedad calcificada de la válvula aórtica con estenosis aortica grave, insuficiencia aórtica, hipertrofia del ventrículo izquierdo e insuficiencia mitral leve. Dentro de los estudios prequirúrgicos presentó VDRL con 1:4 diluciones, se confirmó el diagnóstico con el resultado de la prueba treponémica y se descartaron otras enfermedades infecciosas. Se realizó remplazo valvular con bioprótesis y tubo valvular supracoronario. En la biopsia se confirmó la valvulitis crónica cicatricial con extensas calcificaciones. Posterior a la intervención quirúrgica se dio egreso por adecuada evolución clínica.

Palabras clave: Aortitis. Diagnóstico serológico de la sífilis. Cardiovascular syphilis.

\begin{abstract}
The case of a 73-year-old female patient obtained and resident of Florencia, Caquetá (Colombia), with a history of hypertension who is admitted to a highly complex hospital due to a 3-month clinical picture of constant evolution in pain is presented. Thoracic oppressive type in the anterior region of the left thorax, non-irradiated, of intensity 8/10. The echocardiogram shows calcified aortic valve disease with severe aortic stenosis, aortic regurgitation, left ventricular hypertrophy, and mild grade mitral regurgitation. Within the pre-surgical studies, she presented VDRL with 1:4 dilutions and the diagnosis was confirmed with the result of the treponemal test and other infectious diseases were ruled out. Valvular replacement was performed with a bioprosthesis plus a supra-coronary valvular tube. In the biopsy, chronic scar valvulitis with extensive calcifications was confirmed. After the surgical intervention, discharge was performed due to adequate clinical evolution.
\end{abstract}

Key words: Aortitis. Syphilis serodiagnosis. Syphilis cardiovascular.

\footnotetext{
Correspondencia:

*Diego J. Alvis-Peña

E-mail: diego-927@ hotmail.com

Fecha de recepción: 27-12-2019

Fecha de aceptación: 19-05-2020

DOI: 10.24875/ACM.20000404

Disponible en internet: $13-10-2020$

Arch Cardiol Mex. 2020;90(4):529-534 www.archivoscardiologia.com CC BY-NC-ND (http://creativecommons.org/licenses/by-nc-nd/4.0/).
} 


\section{Introducción}

Siendo la sífilis una enfermedad producida por Treponema pallidum, que se transmite por vía sexual, sanguínea o transplacentaria, caracterizada por sucesivas etapas de evolución, y que hasta el momento su tratamiento con penicilina es bien conocido y eficaz, no debería ser un problema de salud pública, pero actualmente la cifras demuestran lo contrario, pues en Colombia, en el año 2010, fueron diagnosticadas 6754 personas con sífilis, de ellas 836 con diagnóstico de sífilis tardía, y en el año 2011 hubo 992 casos con diagnóstico de sífilis tardía1. La alta proporción en la consulta pone de manifiesto la existencia de barreras para su adecuada clasificación según su estadio, al igual que la falta de oportunidad y de calidad de la atención brindada a los pacientes que consultan por una infección de transmisión sexual ${ }^{2}$.

La sífilis cardiovascular es el sello distintivo de la aortitis, siendo una consecuencia de la sífilis terciaria causada por la infección de la espiroqueta que afecta los medios aórticos con inflamación y posterior cicatrización, produciendo necrosis de la capa media y de igual forma proliferación fibrosa de la túnica íntima ${ }^{3}$. Las manifestaciones clínicas se pueden presentar como aortitis, aneurisma aórtico, insuficiencia aórtica o estenosis de los ostia de las arterias coronarias, siendo la insuficiencia aórtica la complicación más frecuente $^{4}$. Los periodos de latencia son prolongados, entre 10 y 30 años tras la infección primaria, en pacientes que no recibieron tratamiento o con tratamientos incompletos ${ }^{5}$. Aunque la prevalencia de la sífilis cardiovascular posterior a la era de la penicilina ha disminuido relativamente, en los países en vías de desarrollo se siguen reportando casos ${ }^{6}$.

Se han descrito varios casos de complicaciones cardiovasculares tipo aortitis sifilítica, los cuales fueron manejados quirúrgicamente debido a dilatación aneurismática aórtica asociada a insuficiencia aórtica grave, obteniendo resultados favorables ${ }^{4,5}$.

\section{Presentación del caso}

Mujer de 73 años, de etnia mestiza, procedente y residente de Florencia, Caquetá (Colombia), ama de casa. Ingresa al servicio de urgencias de un hospital de alta complejidad de Neiva (Colombia) debido a un cuadro clínico de 3 meses de evolución consistente en dolor torácico tipo opresivo en la región anterior izquierda del tórax, no irradiado, de intensidad 8/10, el cual aumenta con las actividades cotidianas de mediano esfuerzo, que se asocia a disnea progresiva en los últimos 15 días y que mejora con el reposo. Dentro de sus antecedentes de importancia presenta hipertensión arterial, histerectomía hace 15 años y colecistectomía hace 20 años. Niega antecedentes familiares de interés. En tratamiento con metoprolol, amlodipino, ácido acetilsalicílico, losartán, atorvastatina, bisacodilo y alprazolam.

En la exploración física inicial presenta presión arterial de 140/80 mmHg, frecuencia cardiaca de 72 latidos por minuto, frecuencia respiratoria de 18 respiraciones por minuto, pulsioximetría del $92 \%$ al aire ambiente, afebril, peso de $59 \mathrm{~kg}$, hidratada sin ingurgitación venosa yugular, tórax expandible con normalidad, ruidos cardiacos rítmicos con soplo sistólico de grado III audible en todos los focos con predominio en el aórtico, sin presencia de edemas y sistema neurológico sin alteraciones.

El ecocardiograma mostró estenosis aórtica grave con apertura de $0,84 \mathrm{~cm}$ y velocidad máxima de $4.52 \mathrm{~m} / \mathrm{s}$, con insuficiencia aórtica moderada lateralizada hacia la valva anterior de la mitral, hipertrofia concéntrica de las paredes del ventrículo izquierdo por sobrecarga sistólica crónica y dilatación de la aorta ascendente.

La arteriografía coronaria mostró las arterias coronarias sin lesiones y la válvula aórtica con calcificaciones de grado moderado. Se solicitó una angiografía por tomografía computarizada de tórax que confirmó la dilatación aneurismática de la aorta ascendente, de $48 \mathrm{~mm}$ de diámetro (Fig. $1 \mathrm{~A} \mathrm{y} \mathrm{B}$ ).

Las pruebas de laboratorio al ingreso no presentaban alteraciones (hemoglobina $12.6 \mathrm{~g} / \mathrm{dll}$, hematocrito $38.4 \%$; plaquetas $188,100 / \mathrm{mm}^{3}$; tiempo de protombina $17.3 \mathrm{~s}$; tiempo de tromboplastina parcial $39.9 \mathrm{~s}$ ), los electrolitos estaban en rangos normales (sodio $145.6 \mathrm{mmol} / \mathrm{l}$; potasio $4.15 \mathrm{mmol} / \mathrm{l}$ ) y las troponinas eran negativas. Al no presentar alteraciones de laboratorio y por los hallazgos descritos, se programa cirugía cardiovascular para cambio valvular aórtico más tubo supracoronario vs. Bentall. Se solicita el perfil de infección, que reporta baciloscopia sin hallazgo de bacilos acidorresistentes en los 100 campos observados, antígeno de superficie de la hepatitis B 27,18 (positivo), antígeno de superficie de la hepatitis C 27,86 (positivo), anti-HCV IgG 2,32 (positivo), virus de la inmunodeficiencia humana negativo y VDRL (Venereal Disease Research Laboratory) reactiva en 1:4 diluciones. Ante estos hallazgos se suspende cirugía y se determina la carga viral para hepatitis $C$, IgM anticore hepatitis $\mathrm{B}$, antígeno de la hepatitis $\mathrm{B}$, anticuerpos para hepatitis $B$, carga viral para hepatitis $B$ y prueba treponémica; con los resultados del perfil infeccioso se podrán tomar decisiones en cuanto al procedimiento quirúrgico de la paciente. El reporte de la prueba 

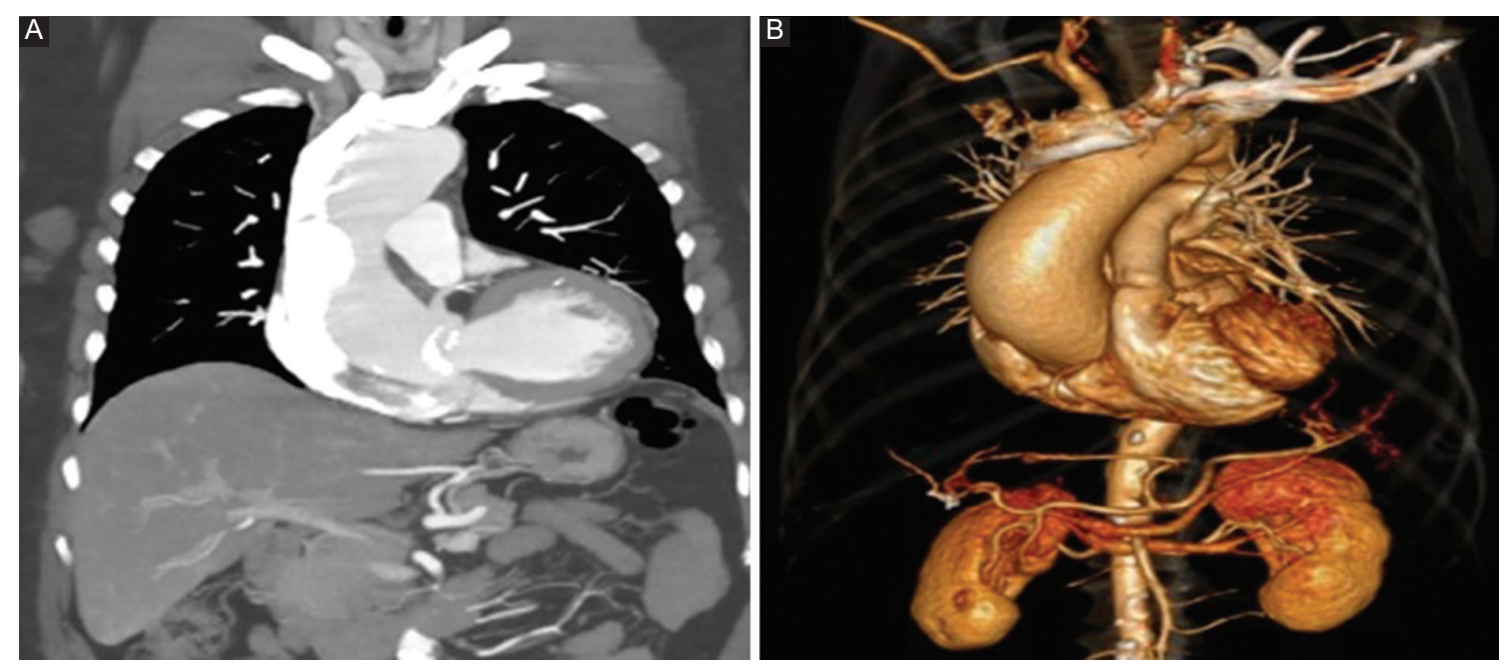

Figura 1. Vista tomográfica A: y reconstrucción tridimensional B: Se evidencia ectasia importante del segmento ascendente de la aorta, con un diámetro de $48 \mathrm{~mm}$, y el diámetro transverso del segmento descendente es de $23 \mathrm{~mm}$, con presencia de placas ateromatosas parietales sin disección de la íntima.
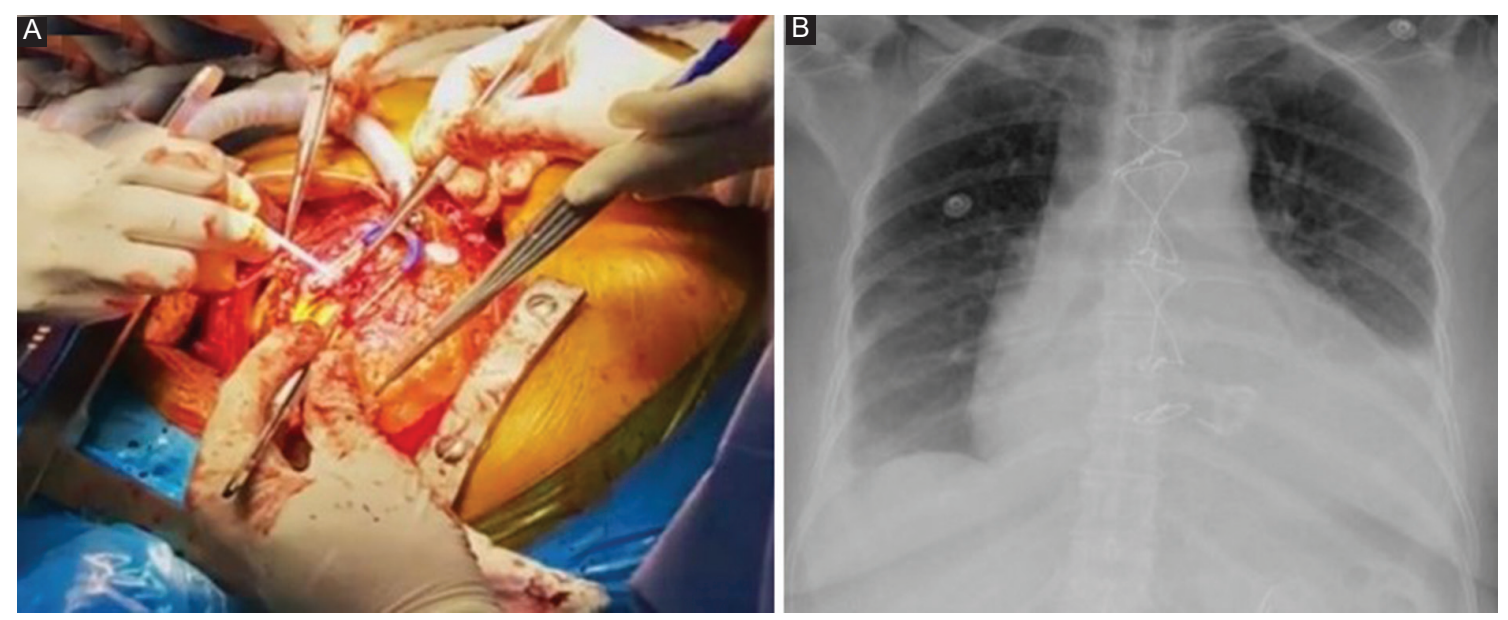

Figura 2. A: Intervención quirúrgica por esternotomía en la que se realiza corrección quirúrgica de sustitución valvular aórtica por bioprótesis Perimount 21 más tuvo supracoronario de $30 \mathrm{~mm}$. B: Radiografía de tórax posquirúrgica.

treponémica fue positivo, por lo que se inició tratamiento con penicilina benzatínica a dosis de 2,400,000 UI por vía intravenosa semanales, por 3 semanas.

Se descartaron las hepatitis $\mathrm{B}$ y $\mathrm{C}$, ya que los resultados de carga viral reportaron ausencia de infección (menos de 10 copias/ $\mathrm{ml}$ para ambas y anticore lgM 0.050 , no reactivo).

Por tratarse de sífilis cardiovascular se realizó tratamiento con penicilina benzatínica y al terminar la última dosis se procedió a la cirugía. La estrategia quirúrgica se discutió teniendo en cuenta los reportes de la tomografía computarizada, el ecocardiograma transtorácico y la angiografía coronaria. Se determinó enviar a la paciente para una sustitucion valvular aórtica por bioprótesis Perimount 21 más tubo supracoronario de $30 \mathrm{~mm}$ (Fig. 2 A y B). La biopsia del material resecado en la cirugía reportó valvulitis crónica cicatricial con extensas calcificaciones. 
El curso posoperatorio de la paciente fue sin ninguna complicación. Luego de su procedimiento quirúrgico se solicitó interconsulta por trabajo social y psicología clínica para evaluar su red de apoyo familiar, la cual fue adecua$\mathrm{da}$, y después de 10 días de posoperatorio con una excelente recuperación se le da de alta con tratamiento de carvedilol (12.5 mg/12 h), amiodarona (20 mg/24 h), losartán $(50 \mathrm{mg} / 12 \mathrm{~h}$ ) y ácido acetilsalicílico (1 mg/24 h).

\section{Discusión}

Presentamos el caso de una mujer de 73 años con sífilis cardiovascular y aortitis con compromiso de la válvula aórtica.

La sífilis es una infección sistémica bacteriana crónica que puede durar décadas, causada por la espiroqueta T. pallidum subespecie pallidum. El único anfitrión y huésped es el ser humano. La presentación de la enfermedad puede variar e imitar diferentes manifestaciones clínicas, por lo que se la conoce como «la gran imitadora». El diagnóstico se basa en la detección serológica lipoidal no específica (RPR [reagina plasmática rápida] y VDRL) seguida de pruebas serológicas específicas treponémicas ${ }^{7,8}$. En todo el mundo se ha observado un reciente aumento de esta enfermedad y su causa es multifactorial, en la que se incluyen una mayor actividad sexual de alto riesgo y sin protección, la migración y los cambios sociales, que disminuyen la oportunidad y la accesibilidad a los servicios de salud para un tratamiento precoz y oportuno. Sin embargo, los regímenes terapéuticos actualmente están bien definidos y son exitosos ${ }^{9}$; como primera línea de tratamiento está la penicilina, que se usó en la paciente antes de la intervención quirúrgica a dosis de 2,400,000 UI por vía intramuscular semanal, por 3 semanas.

La sífilis terciaria puede ocurrir en aproximadamente un tercio de los pacientes no tratados y causar lesiones granulomatosas, llamadas gomas; entre otras manifestaciones se encuentran las cardiovasculares y las neurológicas. Hoy día es raro encontrar este tipo manifestaciones por el éxito de los antibióticos, pero esta infección puede desarrollarse a largo plazo (años y tal vez décadas) después de la infección inicial sin el apropiado manejo, llegando a observarse hasta en el $70 \%$ de los pacientes no tratados ${ }^{4,5,9,10}$.

La aortitis sifilítica es la manifestación cardinal de la sífilis terciaria cardiovascular. Es secundaria a una vasculitis de los vasa vasorum, que genera una endarteritis obliterante con lesión isquémica de la túnica media de la aorta, así como pérdida del músculo liso y de la matriz extracelular. Con mayor frecuencia se ha encontrado compromiso de la aorta torácica ascendente, debido a la significativa cantidad de vasa vasorum y la extensa red linfática de esta región, la cual es invadida por T. pallidum durante la infección primaria. El compromiso excepcional de la aorta torácica se debe a la ausencia de vasa vasorum de la aorta abdominal. Desde otra perspectiva, el debilitamiento de la pared aórtica lleva a una dilatación de la raíz aórtica y a potenciales complicaciones derivadas de ello; las principales son la insuficiencia valvular aórtica (como en nuestra paciente), el aneurisma aórtico sifilítico y la estenosis del ostium de las coronarias ${ }^{10-13}$. Los aneurismas aórticos torácicos crecen hacia arriba a una tasa de $0.10 \mathrm{~cm}$ por año ${ }^{14}$. Las pautas de consenso aconsejan la evaluación quirúrgica independientemente del tamaño para todos los pacientes con síntomas sugestivos de expansión de aneurisma, así como para individuos asintomáticos con un diámetro ascendente del seno aórtico de $5.5 \mathrm{~cm}$ o más ${ }^{15}$. Los aneurismas son complicados debido a la invasión y la erosión de la pared torácica, hecho que complica más el caso en el momento de la intervención quirúrgica.

Como ya se mencionó, la complicación más frecuente es la insuficiencia aórtica, que ocurre en el 20-30\% de los pacientes y es secundaria a una dilatación de la raíz aórtica. Así mismo, hay síntomas de falla cardiaca y es habitual auscultar un soplo en el borde esternal derecho16; en nuestra paciente se evidenció un soplo sistólico de grado III audible en todos los focos y de gran predominio en el foco aórtico. En segunda instancia está el aneurisma sifilítico, el cual es tres veces menos frecuente que la insuficiencia aórtica, detectándose en el 5-10\% de los pacientes; en nuestra paciente se encontró regurgitación aórtica de grado II, calcificación valvular aórtica de grado moderado y aneurisma de la raíz de la aorta y la aorta ascendente, consistente con la literatura sobre la ubicación más habitual de los aneurismas $(50 \%$ en la aorta ascendente, $35 \%$ en el arco aórtico y $15 \%$ en la aorta descendente) $)^{17-20}$.

El diagnóstico de la sífilis cardiovascular se fundamenta en criterios serológicos, histológicos y de imagen, y en nuestra paciente se realizó bajo estas tres premisas. La primera prueba inicial no treponémica se confirmó con las pruebas serológicas treponémicas específicas. Así mismo, se confirmó con el ecocardiograma, la angiografía percutánea y la angiotomografía con reconstrucción tridimensional. En el estudio histológico, la pared arterial de la aorta ascendente se observó con una degeneración de la túnica medica caracterizada por la acumulación de pequeños depósitos 
basaloides sin inflamación y con áreas irregulares y transmurales de desorganización y separación de las fibras. En la pared de la válvula aórtica se observó engrosamiento y esclerosis de la capa esponjosa, con extensas áreas de calcificación. En la superficie ventricular se observó infiltrado inflamatorio crónico subepitelial leve, con erosión focal y agregados de fibrina. No se hallaron granulomas ni microorganismos en el material evaluado. Estos hallazgos son sugestivos de lo encontrado en la literatura por el engrosamiento fibroso de la adventicia con pérdida de los vasa vasorum, rodeado de células linfoplasmocitarias y pérdida de las fibras de elastina en la media, con engrosamiento aterosclerótico de la íntima y generalmente sin evidencia de espiroquetas dentro de la aorta ${ }^{7,8,10}$. En cuanto al tratamiento antibiótico, fue con penicilina por su adecuada sensibilidad y por ser la primera línea terapéutica; se realizó el esquema farmacológico según el régimen establecido por las guías.

El caso presentado ofrece una de las mejores soluciones a pacientes con esta patología y que son de alto riesgo, y puede ser la mejor evidencia de que es una opción de tratamiento disponible para el manejo de esta infección y sus complicaciones potencialmente mortales.

\section{Conclusión}

La sífilis es una infección que continúa siendo prevalente en nuestra población, y por esta razón es muy importante que el personal de salud se familiarice con sus amplias y diferentes manifestaciones clínicas, entre las que se incluyen las presentaciones cardiovasculares, siendo más frecuentes la insuficiencia valvular aórtica, el aneurisma de aorta ascendente y la obstrucción del ostium de las coronarias, que se manifiestan según la evolución natural de la enfermedad en el estadio de sífilis terciaria.

Las enfermedades de transmisión sexual, incluyendo la sífilis, continúan siendo un problema de salud pública en nuestra región y en los países en vías de desarrollo. Si bien la sífilis cardiovascular es una complicación poco frecuente y de larga evolución, el personal de talento humano en salud debe reconocer sus estadios tempranos, sus amplias manifestaciones clínicas y sus factores de riesgo para poder realizar un diagnóstico precoz, teniendo en cuenta las pruebas tanto no treponémicas como treponémicas, un tratamiento oportuno e integral, y así poder llegar a disminuir las posibles complicaciones.

\section{Financiamiento}

La presente investigación no ha recibido ninguna beca específica de agencias de los sectores público, comercial o sin ánimo de lucro.

\section{Conflictos de intereses}

Los autores declaran no tener conflictos de intereses.

\section{Responsabilidades éticas}

Para la realización de la investigación se tuvieron en cuenta los aspectos éticos de la Declaración de Helsinki y la resolución 8430 del Ministerio de Salud de Colombia ${ }^{21,22}$, clasificando esta investigación en un nivel de riesgo mínimo.

Protección de personas y animales. Los autores declaran que para esta investigación no se han realizado experimentos en seres humanos ni en animales.

Confidencialidad de los datos. Los autores declaran que han seguido los protocolos de su centro de trabajo sobre la publicación de datos de pacientes.

Derecho a la privacidad y consentimiento informado. Los autores declaran que en este artículo no aparecen datos de pacientes.

\section{Bibliografía}

1. Ministerio de Salud y Protección Social. Guía de Práctica Clínica para el abordaje sindrómico del diagnóstico y tratamiento de los pacientes con infecciones de transmisión sexual y otras infecciones del tracto genital. Ministerio de Salud y Protección Social - Colciencias de Colombia; 2013. 642 p. Available from: http://gpc.minsalud.gov.co/guias/Pages/Guía-de-infecciones-en-el-tracto-genital.aspx.

2. Turnes AL. La sífilis en la medicina. Una aproximación a su historia. Montevideo, Uruguay: Ediciones Granada; 2007. Disponible en: https:// www.smu.org.uy/publicaciones/libros/textocompleto/la_sifilis_medicina.pdf

3. Xu S, Bendeck M, Gotlieb Al. Vascular pathobiology: atherosclerosis and large vessel disease. En: Buja LM, Butany J, editores. Cardiovascular pathology. Philadelphia: Elsevier; 2016. p. 85-124. Disponible en: https:// www.clinicalkey.es/\#!/content/3-s2.0-B9780124202191000033

4. Uribe-Arango LM, Mejía-Zuluaga M, Bedoya-Jaramillo TM, Arroyave-Carvajal A, Duque-Ramírez M. Aortitis sifilítica: manifestación olvidada de la sífilis. Rev Colomb Cardiol. 2019;26. Disponible en: https://www.clinicalkey.es/\#!/content/1-s2.0-S0120563319301093

5. Figueroa Agudelo FN, Calle Ramírez JA, Gándara Ricardo J, Velásquez O, Muñoz Ortiz E. Aortitis sifilítica: "una vieja olvidada." Rev Colomb Cardiol. 2018;25:236.e1-5. Disponible en: https://www.clinicalkey.es/\#!/content/1-s2.0-S0120563317301316

6. Vaideeswar P. Syphilitic aortitis: rearing of the ugly head. Indian J Pathol Microbiol. 2010;53:624-7.

7. Stamm LV. Syphilis: re-emergence of an old foe. Microbial Cell. 2016;3:363-70.

8. Carrada-Bravo T. Sífilis cardiovascular: diagnóstico y tratamiento. Arch Cardiol Mex. 2006;76(S4):189-96.

9. Byard RW. Syphilis - cardiovascular manifestations of the great imitator. J Forensic Sci. 2018;63:1312-5.

10. Blome A, Isenberg D. Aortic thrombus in a case of tertiary syphilis. J Emerg Med. 2019;56:564-5.

11. Roberts WC, Ko JM, Vowels TJ. Natural history of syphilitic aortitis. Am J Cardiol. 2009;104:1578-87. Disponible en: http://dx.doi.org/10.1016/j. amjcard.2009.07.031 
12. De Almeida Feitosa IN, Dantas Leite Figueiredo M, de Sousa Belem L, Evelin Soares Filho AW. Rare presentation of ruptured syphilitic aortic aneurysm with pseudoaneurysm. Rev Port Cardiol (English ed). 2015;34:691. e1-4. Disponible en: $h$ ttp://dx.doi.org/10.1016/.repce.2015.10.002

13. Roberts WC, Barbin CM, Weissenborn MR, Ko JM, Henry AC. Syphilis as a cause of thoracic aortic aneurysm. Am J Cardiol. 2015;116:1298303. Disponible en: http://dx.doi.org/10.1016/i.amjcard.2015.07.030

14. Coady MA, Rizzo JA, Hammond GL, Kopf GS, Elefteriades JA. Surgica intervention criteria for thoracic aortic aneurysms: a study of growth rates and complications. Ann Thorac Surg. 1999;67:1922-6.

15. Hiratzka LF, Bakris GL, Beckman JA, Bersin RM, Carr VF, Casey DE, et al. 2010 ACCF/AHA/AATS/ACR/ASA/SCA/SCAI/SIR/STS/SVM guidelines for the diagnosis and management of patients with thoracic aortic disease: Executive summary: a report of the American College of Cardiology Foundation/American Heart Association Task Force on Practice Guidelines, American Association for Thoracic Surgery, American College of Radiology, American Stroke Association. Circulation. 2010;121:266-369.

16. Roberts WC, Moore AJ, Roberts CS. Syphilitic aortitis: still a current common cause of aneurysm of the tubular portion of ascending aorta.
Cardiovasc Pathol. 2019;107175. Disponible en: https://doi.org/10.1016/j. carpath.2019.107175

17. Yuan S-M. Syphilitic aortic aneurysm. Z Für Rheumatol. 2018;77:741. 8.12

18. Girardi LN. Syphilitic aortitis: the bigger picture. J Thorac Cardiovasc Surg. 2017;154:e27-8. Disponible en: http://dx.doi.org/10.1016/j.jcvs.2017.04.052

19. Pivatto Júnior F, Finkler BS, Torres FS, Schaefer PG, Sprinz E. Aneurysm and dissection in a patient with syphilitic aortitis. Brazilian $J$ Infect Dis. 2017;21:349-52. Disponible en: http://dx.doi.org/10.1016/j. bjid.2017.01.003

20. Tennant E, Post JJ, Overton K, Gulholm T, McKenzie P. A case of syphilitic aortitis. Intern Med J. 2018;48:1549-50.

21. Di Ruggiero M. Declaración de Helsinki, principios y valores bioéticos en juego en la investigación médica con seres humanos. Revista Colombiana de Bioética. 2011;6:125-44.

22. Ministerio de Salud. Resolución N.o 008430 de 1993, Octubre 4, por la cual se establecen las normas científicas, técnicas y administrativas para la investigación en salud. 1993. 